University of New Orleans

ScholarWorks@UNO

8-2004

\title{
Phase shifts that accompany total internal reflection at a dielectric-dielectric interface
}

R. M.A. Azzam

University of New Orleans, razzam@uno.edu

Follow this and additional works at: https://scholarworks.uno.edu/ee_facpubs

Part of the Electrical and Electronics Commons, and the Optics Commons

\section{Recommended Citation}

R. M. A. Azzam, "Phase shifts that accompany total internal reflection at a dielectric-dielectric interface," J. Opt. Soc. Am. A 21, 1559-1563 (2004)

This Article is brought to you for free and open access by the Department of Electrical Engineering at ScholarWorks@UNO. It has been accepted for inclusion in Electrical Engineering Faculty Publications by an authorized administrator of ScholarWorks@UNO. For more information, please contact scholarworks@uno.edu. 


\title{
Phase shifts that accompany total internal reflection at a dielectric-dielectric interface
}

\author{
R. M. A. Azzam \\ Department of Electrical Engineering, University of New Orleans, New Orleans, Louisiana 70148
}

Received January 13, 2004; revised manuscript received March 18, 2004, accepted March 23, 2004

\begin{abstract}
The absolute, average, and differential phase shifts that $p$ - and $s$-polarized light experience in total internal reflection (TIR) at the planar interface between two transparent media are considered as functions of the angle of incidence $\phi$. Special angles at which quarter-wave phase shifts are achieved are determined as functions of the relative refractive index $N$. When the average phase shift equals $\pi / 2$, the differential reflection phase shift $\Delta$ is maximum, and the reflection Jones matrix assumes a simple form. For $N>\sqrt{3}$, the average and differential phase shifts are equal (hence $\delta_{p}=3 \delta_{s}$ ) at a certain angle $\phi$ that is determined as a function of $N$. All phase shifts rise with infinite slope at the critical angle. The limiting slope of the $\Delta$-versus- $\phi$ curve at grazing incidence $(\partial \Delta / \partial \phi)_{\phi=90^{\circ}}=-(2 / N)\left(N^{2}-1\right)^{1 / 2}=-2 \cos \phi_{c}$, where $\phi_{c}$ is the critical angle and $\left(\partial^{2} \Delta / \partial \phi^{2}\right)_{\phi=90^{\circ}}=0$. Therefore $\Delta$ is proportional to the grazing incidence angle $\theta=90^{\circ}-\phi$ (for small $\theta$ ) with a slope that depends on $N$. The largest separation between the angle of maximum $\Delta$ and the critical angle is $9.88^{\circ}$ and occurs when $N=1.55377$. Finally, several techniques are presented for determining the relative refractive index $N$ by using TIR ellipsometry. (C) 2004 Optical Society of America
\end{abstract}

OCIS codes: 240.0240, 260.0260, 260.2130, 260.5430, 260.6970.

\section{INTRODUCTION}

The reflection of $p$ - and $s$-polarized light at the planar interface between two semi-infinite, homogeneous, isotropic media is governed by the well-known Fresnel coefficients. $^{1-3}$ When both media are transparent, the phase shifts that accompany partial external or internal reflection assume the values of 0 or $\pi$. However, under conditions of total internal reflection (TIR), at angles of incidence $\phi$ above the critical angle

$$
\phi_{c}=\arcsin n,
$$

the phase shifts $\delta_{p}, \delta_{s}$, and $\Delta=\delta_{p}-\delta_{s}$ are nontrivial and are given $\mathrm{by}^{2,3}$

$$
\begin{aligned}
& \tan \left(\delta_{p} / 2\right)=N\left(N^{2} \sin ^{2} \phi-1\right)^{1 / 2} / \cos \phi, \\
& \tan \left(\delta_{s} / 2\right)=\left(N^{2} \sin ^{2} \phi-1\right)^{1 / 2} /(N \cos \phi), \\
& \tan (\Delta / 2)=\left(N^{2} \sin ^{2} \phi-1\right)^{1 / 2} /(N \sin \phi \tan \phi) .
\end{aligned}
$$

In Eqs. (1)-(4),

$$
N=N_{0} / N_{1}
$$

is the high-to-low ratio of refractive indices of the media of incidence and (evanescent) refraction, respectively $(N$ $>1$ ), and $n=1 / N<1$.

In addition to the differential reflection phase shift $\Delta$ (which is measurable by ellipsometry ${ }^{3}$ ), we introduce the average phase shift on reflection

$$
\delta_{a}=\left(\delta_{p}+\delta_{s}\right) / 2
$$

Based on Eqs. (2) and (3) and the trigonometric identity for the tangent of the sum of two angles, we obtain

$$
\begin{aligned}
\tan \delta_{a}= & {\left[(\cos \phi)\left(N+N^{-1}\right)\left(N^{2} \sin ^{2} \phi-1\right)^{1 / 2}\right] / } \\
& {\left[2-\left(\sin ^{2} \phi\right)\left(N^{2}+1\right)\right] . }
\end{aligned}
$$

From Eqs. (2) and (3), we also obtain the following useful relation between $\delta_{p}$ and $\delta_{s}$,

$$
\tan \left(\delta_{p} / 2\right)=N^{2} \tan \left(\delta_{s} / 2\right),
$$

which is valid for any interface (a given $N$ ) at any angle of incidence in the range $\phi_{c} \leqslant \phi \leqslant 90^{\circ}$. Another direct relation between $\delta_{p}$ and $\delta_{s}$ that is valid at a given $\phi$ independent of $N$ was previously published. ${ }^{4}$

In this paper we derive a number of interesting new results concerning the TIR phase shifts $\delta_{p}, \delta_{s}, \delta_{a}$, and $\Delta$. In Section 2 we find the angles of incidence at which these phase shifts are quarter-wave $(=\pi / 2)$ and discuss their significance. Analogous to the Abelès condition, ${ }^{5} \delta_{p}$ $=2 \delta_{s}$ at $\phi=45^{\circ}$, we investigate the condition $\delta_{a}=\Delta$, which is equivalent to $\delta_{p}=3 \delta_{s}$, in Section 3 . In Section 4 the limiting slopes of the $\Delta$-versus- $\phi$ curve at the critical angle and at grazing incidence are obtained. In Section 5 , the difference between the angle of incidence $\phi_{m}$ of maximum $\Delta$ and the critical angle $\phi_{c}$ is obtained as a function of $N$, and it is shown that this difference $\phi_{m}$ - $\phi_{c}$ cannot exceed $9.88^{\circ}$. In Section 6 we propose several methods for recovering $N$ based on various features of the $\Delta$-versus- $\phi$ curve. Section 7 is a brief summary of the paper.

\section{QUARTER-WAVE PHASE SHIFTS IN TOTAL INTERNAL REFLECTION}

As a specific example, Fig. 1 shows the phase shifts $\delta_{p}$, $\delta_{s}, \delta_{a}$, and $\Delta$ on TIR at the glass-air interface $(N$ 


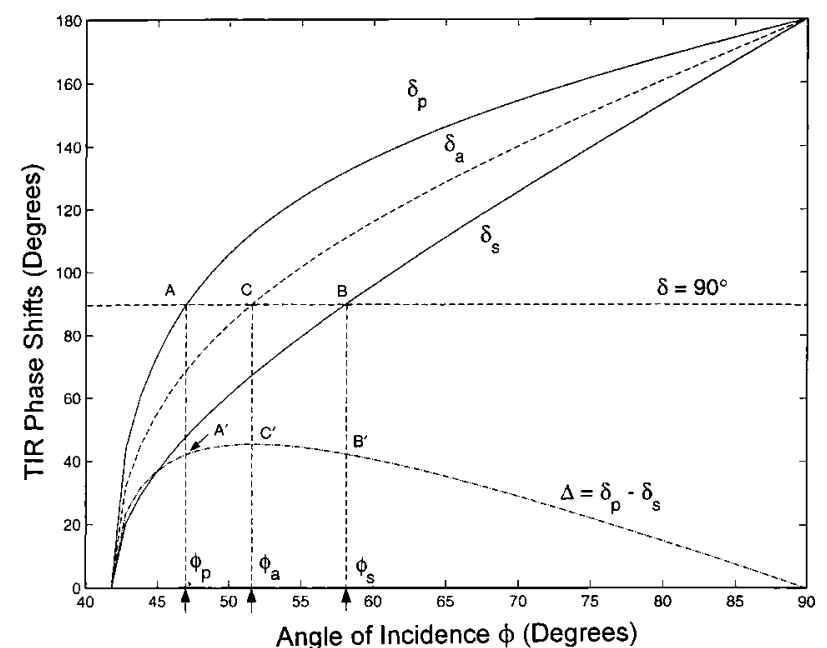

Fig. 1. TIR phase shifts $\delta_{p}, \delta_{s}, \delta_{a}$, and $\Delta$ at the glass-air interface $(N=1.5)$ plotted as functions of the angle of incidence $\phi$ between the critical angle $\phi_{c}=\arcsin (2 / 3)=41.81^{\circ}$ and grazing incidence $\phi=90^{\circ}$. Quarter-wave phase shifts are attained at points $\mathrm{A}, \mathrm{B}$, and $\mathrm{C}$ where the line $\delta=90^{\circ}$ intersects the curves of $\delta_{p}, \delta_{s}$, and $\delta_{a}$ at angles of incidence denoted by $\phi_{p}, \phi_{s}$, and $\phi_{a}$, respectively.

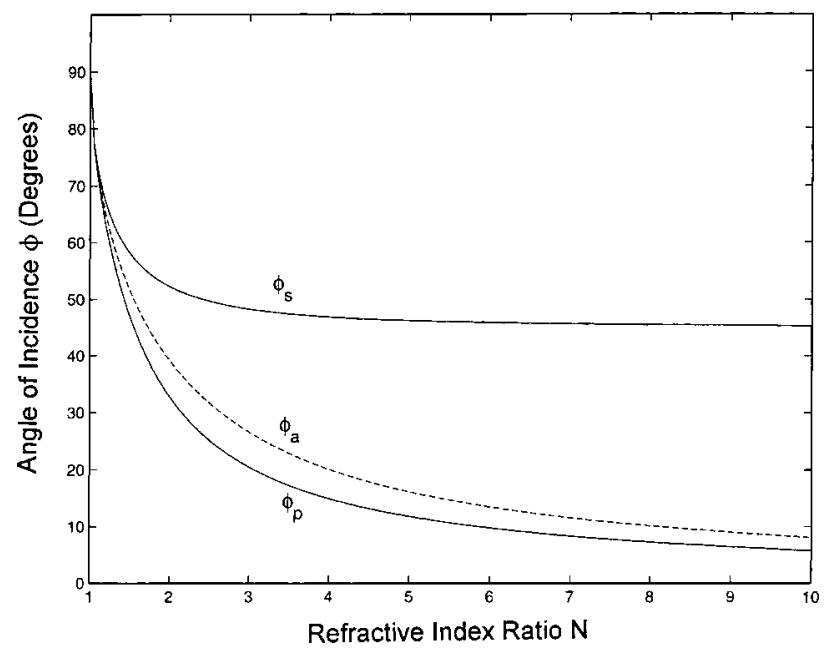

Fig. 2. Three angles of incidence $\phi_{p}, \phi_{s}$, and $\phi_{a}$, at which the $p, s$, and average TIR phase shifts [Eqs. (9)-(11)] are quarterwave are plotted as functions of the refractive index ratio $N$.

$=1.5)$ plotted as functions of the angle of incidence $\phi$ between the critical angle $\phi_{c}=\arcsin (2 / 3)=41.81^{\circ}$ and grazing incidence $\phi=90^{\circ}$. Quarter-wave phase shifts are attained at points $\mathrm{A}, \mathrm{B}$, and $\mathrm{C}$ where the line $\delta$ $=90^{\circ}$ intersects the curves of $\delta_{p}, \delta_{s}$, and $\delta_{a}$ at angles of incidence denoted by $\phi_{p}, \phi_{s}$, and $\phi_{a}$, respectively.

Expressions for the angles $\phi_{p}$ and $\phi_{s}$ for any $N$ are readily obtained by setting the left-hand sides of Eqs. (2) and (3) equal to 1 . The corresponding expression for $\phi_{a}$ is obtained by setting the denominator of the right-hand side of Eq. (7) equal to 0. The results are listed below:

$$
\begin{aligned}
& \sin ^{2} \phi_{p}=\left(N^{2}+1\right) /\left(N^{4}+1\right), \\
& \sin ^{2} \phi_{s}=\left(N^{2}+1\right) /\left(2 N^{2}\right), \\
& \sin ^{2} \phi_{a}=2 /\left(N^{2}+1\right) .
\end{aligned}
$$

Figure 2 shows the three angles $\phi_{p}, \phi_{s}$, and $\phi_{a}$ plotted as functions of $N$. All angles approach $90^{\circ}$ as $N$ tends to 1 and decrease monotonically as $N$ is increased. For large $N, \phi_{s}$ approaches $45^{\circ}$, whereas $\phi_{p}$ and $\phi_{a}$ approach 0 .

It will be shown in Section 4 that the angle $\phi_{a}$ for quarter-wave average phase shift given by Eq. (11) is exactly the same as the angle $\phi_{m}$ at which $\Delta$ is maximum [see Eq. (26) below]. (In Fig. 1 the peak point $\mathrm{C}^{\prime}$ of the $\Delta$-versus- $\phi$ curve lies vertically below point C.) Curiously, this also happens to be the angle of incidence at which $p$ - and $s$-polarized light tunnel equally across a thin uniform air gap between two half-prisms of the same refractive index under conditions of frustrated TIR. ${ }^{6-8}$

It is also of interest to calculate $\delta_{s}$ when $\delta_{p}=\pi / 2$, and $\delta_{p}$ when $\delta_{s}=\pi / 2$. The results are readily obtained from Eq. (8):

$$
\begin{array}{ll}
\tan \left(\delta_{s} / 2\right)=1 / N^{2}, & \delta_{p}=\pi / 2, \\
\tan \left(\delta_{p} / 2\right)=N^{2}, & \delta_{s}=\pi / 2 .
\end{array}
$$

Consequently, the differential reflection phase shifts at $\phi_{p}$ and $\phi_{s}$ are

$$
\begin{aligned}
& \Delta\left(\phi_{p}\right)=(\pi / 2)-2 \arctan \left(1 / N^{2}\right), \\
& \Delta\left(\phi_{s}\right)=2 \arctan \left(N^{2}\right)-(\pi / 2) .
\end{aligned}
$$

From Eqs. (13) it follows that

$$
\Delta\left(\phi_{p}\right)=\Delta\left(\phi_{s}\right)
$$

The equal differential reflection phase shifts are represented by points $\mathrm{A}^{\prime}$ and $\mathrm{B}^{\prime}$ in Fig. 1 .

The maximum differential reflection phase shift (at point $\mathrm{C}^{\prime}$ ) is given by ${ }^{9}$

$$
\tan \left(\Delta_{\max } / 2\right)=\left(N^{2}-1\right) /(2 N) .
$$

When $N=1.5, \Delta_{\max }=45.24^{\circ}$ and the $\Delta$-versus- $\phi$ curve lies entirely below the $\delta=90^{\circ}$ line in Fig. 1. To achieve quarter-wave differential phase shift on single reflection (i.e., $\Delta=90^{\circ}$ ), we must have $N \geqslant \sqrt{2}+1=2.414$, according to Eq. (15).

Figure 3 is similar to Fig. 1 except that now $N=4$, which corresponds to the Ge-air interface in the infrared. The Abelès condition, ${ }^{5} \Delta=\delta_{s}$, or $\delta_{p}=2 \delta_{s}$ at $\phi=45^{\circ}$, is

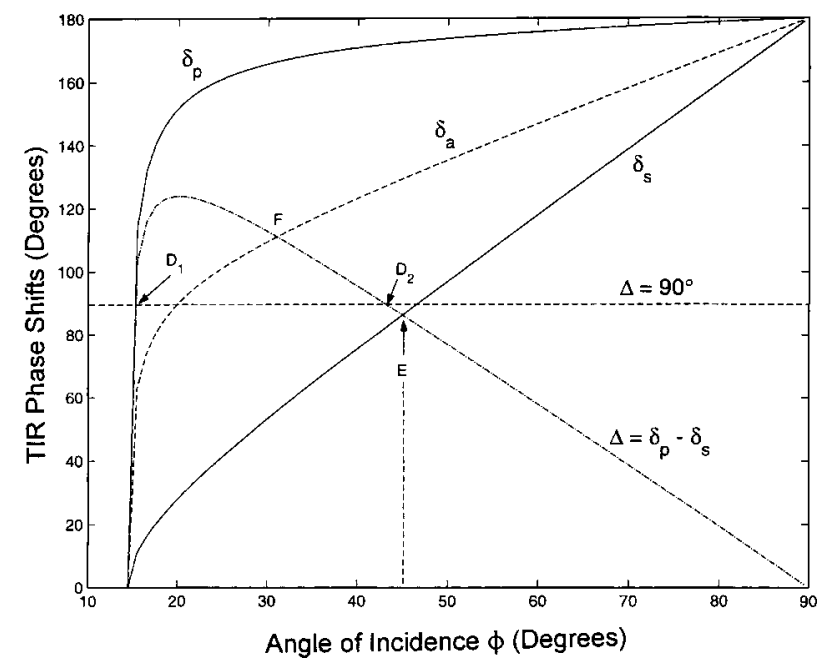

Fig. 3. As in Fig. 1 except that $N=4$, which corresponds to the Ge-air interface in the infrared. The significance of the marked points $\mathrm{D}_{1}, \mathrm{D}_{2}, \mathrm{E}$, and $\mathrm{F}$ is discussed in the text. 
satisfied at point $\mathrm{E}$ in Fig. 3. (The corresponding point at $\phi=45^{\circ}$ in Fig. 1 is not marked.) Figure 3 also clearly shows that $\Delta=90^{\circ}$ is now possible at the two points marked $\mathrm{D}_{1}$ and $\mathrm{D}_{2}$. The corresponding angles of incidence $\phi_{1}$ and $\phi_{2}$ are determined by ${ }^{10}$

$$
\sin ^{2} \phi_{1,2}=\left(\frac{1}{4}\right)\left[\left(n^{2}+1\right) \mp\left(n^{4}-6 n^{2}+1\right)^{1 / 2}\right],
$$

where $n=1 / N$, as before. For the Ge-air interface $n$ $=0.25$, and Eq. (16) gives $\phi_{1}=15.04^{\circ}$ (which is slightly above the critical angle $\phi_{c}=14.48^{\circ}$ ) and $\phi_{2}=42.93^{\circ}$. A Ge, single-reflection, quarter-wave retarder can be designed to operate at $\phi_{2}=42.93^{\circ}$ (point $\mathrm{D}_{2}$ in Fig. 3) with acceptable angular sensitivity. Standard silica-based Fresnel rhomb quarter-wave retarders use 2, 3, or 4 TIRs. ${ }^{11}$

At the angle of incidence $\phi_{m}=\phi_{a}$, where $\Delta=\Delta_{\max }$, we can solve the following two equations:

$$
\begin{aligned}
& \left(\delta_{p} / 2\right)+\left(\delta_{s} / 2\right)=\pi / 2, \\
& \left(\delta_{p} / 2\right)-\left(\delta_{s} / 2\right)=\Delta_{\max } / 2,
\end{aligned}
$$

to obtain $\delta_{p}$ and $\delta_{s}$ in terms of $\Delta_{\max }$ only:

$$
\begin{aligned}
& \delta_{p}=\left(\pi+\Delta_{\max }\right) / 2, \\
& \delta_{s}=\left(\pi-\Delta_{\max }\right) / 2 .
\end{aligned}
$$

Consequently, the reflection Jones matrix ${ }^{3}$ at $\phi_{m}=\phi_{a}$ takes the simple form

$$
R=j\left[\begin{array}{cc}
\exp \left(j \Delta_{\max } / 2\right) & 0 \\
0 & \exp \left(-j \Delta_{\max } / 2\right)
\end{array}\right] .
$$

The outside multiplier $j$ in Eq. (19) represents the quarter-wave average phase shift on reflection.

\section{EQUAL AVERAGE AND DIFFERENTIAL PHASE SHIFTS}

At the point $\mathrm{F}$ where the $\delta_{a}$ and $\Delta$-versus- $\phi$ curves intersect in Fig. 3, the average and differential reflection phase shifts are equal,

$$
\begin{aligned}
\delta_{a} & =\Delta, \\
\left(\delta_{p}+\delta_{s}\right) / 2 & =\delta_{p}-\delta_{s}, \\
\delta_{p} & =3 \delta_{s} .
\end{aligned}
$$

Equations (20), which are to our knowledge new, bear some resemblance to the Abelès condition, ${ }^{5} \Delta=\delta_{s}$, or $\delta_{p}=2 \delta_{s}$, which occurs at $\phi=45^{\circ}$. However, the angle of incidence at which Eq. (20) is satisfied is a function of $N$ given by

$$
\sin ^{2} \phi=\left(N^{2}+1\right) /\left(4 N^{2}\right) .
$$

The derivation leading to Eq. (21), starting from Eqs. (4) and (7), is a bit lengthy and requires some patience, so it is omitted here to save space. Equation (21) has an acceptable solution for $\phi$ only if

$$
\sin ^{2} \phi \geqslant \sin ^{2} \phi_{c}=1 / N^{2} \text {. }
$$

Based on Eqs. (21) and (22), an acceptable nontrivial solution $^{12}$ of Eq. (20) exists only if

$$
N>\sqrt{3}=1.732 \text {. }
$$

For the Ge-air interface $N=4$, and Eq. (21) gives $\phi$ $=31.02^{\circ}$, which precisely locates the point $\mathrm{F}$ in Fig. 3.

\section{LIMITING SLOPES OF THE $\Delta$-versus- $\phi$ CURVE AT THE CRITICAL ANGLE AND GRAZING INCIDENCE}

By taking the derivative of both sides of Eq. (4) with respect to $\phi$, we obtain

$$
\begin{aligned}
\frac{1}{2}\left[\sec ^{2}(\Delta / 2)\right](\partial \Delta / \partial \phi)= & {\left[\left(1-N^{2}\right) \tan ^{2} \phi+2\right] / } \\
& {\left[N \sin \phi \tan ^{2} \phi\right.} \\
& \left.\times\left(N^{2} \sin ^{2} \phi-1\right)^{1 / 2}\right] .
\end{aligned}
$$

The maximum differential reflection phase shift $\Delta$ occurs at the angle $\phi_{m}$ where $\partial \Delta / \partial \phi=0$. By setting the numerator of the right-hand side of Eq. (24) equal to 0, we get

$$
\begin{aligned}
\tan ^{2} \phi_{m} & =2 /\left(N^{2}-1\right), \\
\sin ^{2} \phi_{m} & =2 /\left(N^{2}+1\right) .
\end{aligned}
$$

Equations (11) and (26) lead to the conclusion that $\phi_{m}$ $=\phi_{a}$, as has already been noted in Section $2 .{ }^{13}$

At the critical angle, $N^{2} \sin \phi=1$, and the slope of the $\Delta$-versus- $\phi$ curve is infinite,

$$
(\partial \Delta / \partial \phi)_{\phi=\phi_{c}}=\infty,
$$

as can be seen from Eq. (24). Similarly, by differentiation of Eqs. (2) and (3), it can also be verified that

$$
\left(\partial \delta_{p} / \partial \phi\right)_{\phi=\phi_{c}}=\left(\partial \delta_{s} / \partial \phi\right)_{\phi=\phi_{c}}=\infty .
$$

Therefore all phase shifts rise vertically with respect to $\phi$ at the critical angle. (The angular resolution used in plotting Figs. 1 and 3 does not make this clear.)

The limiting slope of the $\Delta$-versus- $\phi$ curve at grazing incidence $\left(\phi=90^{\circ}\right)$ is also obtained from Eq. (24) as

$$
\begin{aligned}
(\partial \Delta / \partial \phi)_{\phi=90^{\circ}} & =-(2 / N)\left(N^{2}-1\right)^{1 / 2} \\
& =-2\left(1-n^{2}\right)^{1 / 2}=-2 \cos \phi_{c} .
\end{aligned}
$$

For large $N$, the limiting slope of the $\Delta$-versus- $\phi$ curve at grazing incidence in TIR approaches -2 . For the Ge-air interface $(N=4)$, the limiting slope equals -1.9365 .

We have also verified analytically that the second derivative of $\Delta$ with respect to $\phi$ at grazing incidence is zero,

$$
\left(\partial^{2} \Delta / \partial \phi^{2}\right)_{\phi=90^{\circ}}=0 .
$$

Therefore, $\Delta$ is nearly a linear function of $\phi$ near grazing incidence, as can be readily inferred from Figs. 1 and 3. In terms of the grazing incidence angle

$$
\theta=90^{\circ}-\phi,
$$

$\Delta$ can be expressed as

$$
\Delta \approx 2\left(1-n^{2}\right) \theta .
$$

The approximation in Eq. (32) is good for small $\theta$, and gets better as $N$ increases. For example, when $n=0.25(N$ $=4$ ) and $\theta=10^{\circ}$, Eq. (32) gives $\Delta=19.365^{\circ}$, which is only $0.008^{\circ}$ above the exact value predicted by Eq. (4).

Equation (32) has potential application for describing changes in the state of polarization of light that travels as 
paraxial rays in cladless high-index fibers. In an ellipsometer, ${ }^{3}$ Eq. (32) establishes a link between the angle of incidence and the azimuth settings of the polarizing elements that determine $\Delta$.

\section{SEPARATION BETWEEN THE CRITICAL ANGLE AND THE ANGLE OF MAXIMUM DIFFERENTIAL REFLECTION PHASE SHIFT}

Consider the difference

$$
\Delta \phi=\phi_{m}-\phi_{c},
$$

between the angle of maximum differential phase shift and the critical angle. By taking the sine of both sides of Eq. (33),

$$
\sin \Delta \phi=\sin \phi_{m} \cos \phi_{c}-\cos \phi_{m} \sin \phi_{c},
$$

and making use of $\sin \phi_{c}=1 / N$ and $\sin \phi_{m}=\sqrt{2} /\left(N^{2}\right.$ $+1)^{1 / 2}$, we obtain

$$
\sin (\Delta \phi)=(\sqrt{2}-1)\left(N^{2}-1\right)^{1 / 2} /\left[N\left(N^{2}+1\right)^{1 / 2}\right] .
$$

Equation (35) gives the separation of the angle of maximum differential reflection phase shift and the critical angle as a function of the relative refractive index $N .{ }^{14}$

From Eq. (35) note that the difference $\Delta \phi \rightarrow 0$ in both limits as $N \rightarrow 1, N \rightarrow \infty$. Therefore, $\Delta \phi$ must reach a maximum at a certain value of $N$. By equating the derivative of Eq. (35) to 0, the refractive index for which $\Delta \phi$ is maximum is obtained as

$$
N=(\sqrt{2}+1)^{1 / 2}=1.55377 \text {. }
$$

This refractive index is that of a dense glass. The maximum separation is given by Eq. (35) as

$$
\sin (\Delta \phi)_{\max }=(\sqrt{2}-1)^{2}, \quad(\Delta \phi)_{\max }=9.879^{\circ} .
$$

For completeness, we also note that $\Delta_{\max }=48.94^{\circ}$ when $N=1.55377$.

The double-reflection, Fresnel rhomb ${ }^{2,11}$ can be designed to operate under the condition of minimum angular sensitivity by selecting

$$
\Delta=\Delta_{\max }=45^{\circ} .
$$

Substitution of this value into Eq. (15) gives the optimum refractive index,

$$
N=1.49661 \text {, }
$$

of the material (glass) from which the rhomb is made. From Eq. (15), the operating angle of incidence is calculated, $\phi_{m}=51.785^{\circ}$. The separation between this angle and the critical angle, $\Delta \phi=9.858^{\circ}$, is very close to maximum.

\section{MULTIPLE DETERMINATIONS OF THE RELATIVE REFRACTIVE INDEX}

Here we summarize several possible techniques for the determination of the relative refractive index $N$ or $n$.
1. A well-known method is to measure the critical angle $\phi_{c}$ for the onset of TIR and use

$$
n=\sin \phi_{c} \text {. }
$$

2. From ellipsometric ${ }^{3,15}$ measurement of $\Delta$ at a known angle of incidence $\phi$ above the critical angle, Eq. (4) can be inverted to obtain $N$ or $n$ :

$$
n^{2}=\sin ^{2} \phi\left[1-\tan ^{2} \phi \tan ^{2}(\Delta / 2)\right] .
$$

From Eq. (40) we note that since $n>0$, the following relation,

$$
\tan \phi \tan (\Delta / 2)<1,
$$

must be satisfied at all angles of TIR. Inequality (41) can be recast in the equivalent form

$$
\Delta<2\left(90^{\circ}-\phi\right)=2 \theta .
$$

This provides yet another interesting relation concerning $\Delta$ under conditions of TIR.

3. Measurement of the maximum differential reflection phase shift $\Delta_{\max }$ allows $N$ and $n$ to be determined by Eq. (15):

$$
\begin{gathered}
N=\sec \left(\Delta_{\max } / 2\right)+\tan \left(\Delta_{\max } / 2\right), \\
n=\sec \left(\Delta_{\max } / 2\right)-\tan \left(\Delta_{\max } / 2\right) .
\end{gathered}
$$

It is interesting to note that this method does not require that the angle of incidence be measured.

4. Measurement of the angle of incidence of maximum differential reflection phase shift $\phi_{m}$ permits direct determination of $N$ by use of Eq. (26):

$$
N^{2}=2 \csc ^{2} \phi_{m}-1 \text {. }
$$

5. Finally, measurement of the slope of the $\Delta$-versus- $\phi$ curve near grazing incidence also permits $n$ to be determined from Eq. (29):

$$
n^{2}=1-0.25\left[(\partial \Delta / \partial \phi)_{\phi=90^{\circ}}\right]^{2} .
$$

The extent to which these different methods yield consistent values of $N$ can be used to assess the precision and accuracy of a given ellipsometer arrangement.

\section{SUMMARY}

We have presented numerous new results concerning the absolute, average, and differential phase shifts that $p$ and $s$-polarized light experience in total internal reflection (TIR) at the planar interface between two transparent media. Angles of incidence for which quarter-wave phase shifts are achieved in TIR are determined as functions of the relative refractive index $N$. When the average phase shift $\delta_{a}=\left(\delta_{p}+\delta_{s}\right) / 2=\pi / 2$, the differential reflection phase shift $\Delta=\delta_{p}-\delta_{s}$ is maximum, and the reflection Jones matrix assumes the simple form given by Eq. (19).

For interfaces between transparent media with $N$ $>\sqrt{3}=1.732$, the average and differential phase shifts are equal (hence $\delta_{p}=3 \delta_{s}$ ) at an angle of incidence given by Eq. (21).

We also find that all phase shifts rise with infinite slope at the critical angle. The limiting slope of the $\Delta$-versus- $\phi$ 
curve at grazing incidence $\left(\phi=90^{\circ}\right)$ is negative and its absolute value equals double the cosine of the critical angle [Eq. (29)]. Near grazing incidence, the differential reflection phase shift $\Delta$ is approximately proportional to the grazing incidence angle with proportionality constant that depends on $N$ [Eq. (32)].

The difference $\Delta \phi=\phi_{m}-\phi_{c}$ between the angle of maximum $\Delta$ and the critical angle is determined as a function of $N$ [Eq. (35)]. This difference is maximum $\left[(\Delta \phi)_{\max }=\arcsin (\sqrt{2}-1)^{2}=9.8793^{\circ}\right] \quad$ when $N$ $=1.55377$.

Finally, several techniques are presented for determining the relative refractive index $N$ by TIR ellipsometry [Section 6].

R. M. A. Azzam can be reached by e-mail at razzam @uno.edu.

\section{REFERENCES AND NOTES}

1. A. Fresnel, "Mémoire sur la loi des modifications que la réflexion imprime à la lumière polarisée," in Ouvres Complète de Fresnel, Vol. 1, H. Senarmont, E. Verdet, and A. Fresnel, 1866, pp. 767-775 (Johnson Reprint Corporation, New York, 1965).

2. M. Born and E. Wolf, Principles of Optics (Pergamon, New York, 1975).

3. R. M. A. Azzam and N. M. Bashara, Ellipsometry and Polarized Light (North-Holland, Amsterdam, 1987).

4. R. M. A. Azzam, "Relations between amplitude reflectances and phase shifts of the $p$ and $s$ polarizations when electromagnetic radiation strikes interfaces between transparent media," Appl. Opt. 18, 1884-1886 (1979).

5. F. Abelès, "Un théoreme relatif à la réflexion métallique," $\mathrm{C}$ R. Hebd. Seances Acad. Sci. 230, 1942-1943 (1950).

6. E. E. Hall, "The penetration of totally reflected light into the rarer medium," Phys. Rev. 15, 73-106 (1902).

7. P. W. Baumeister, "Optical tunneling and its application to optical filters,” Appl. Opt. 6, 897-905 (1967).

8. R. W. Astheimer, G. Falbel, and S. Minkowitz, "Infrared modulation by means of frustrated total internal reflection," Appl. Opt. 5, 87-91 (1966).

9. See Ref. 2 , p. 50 .

10. R. M. A. Azzam, "Contours of constant principal angle and constant principal azimuth in the complex $\epsilon$ plane," J. Opt. Soc. Am. 71, 1523-1528 (1981).

11. J. Bennett and H. E. Bennett, "Polarization," in Handbook of Optics, W. G. Driscoll and W. Vaughan, eds. (McGraw Hill, New York, 1978), Sect. 10.

12. For any $N$, Eq. (20) also has a trivial solution at $\phi_{c}$, where all phase shifts are zero.

13. From Eq. (26) we also obtain $\cos ^{2} \phi_{m}=\left(N^{2}-1\right) /\left(N^{2}+1\right)$ $=-\cos \left(2 \phi_{B}\right)$, which provides a direct relation between the incidence angle of maximum TIR differential phase shift $\phi_{m}$ and the Brewster angle of external reflection $\phi_{B}$.

14. From Eqs. (35) and (26) it is readily verified that $\sin ^{2}(\Delta \phi)<\sin ^{2}\left(90^{\circ}-\phi_{m}\right)=\cos ^{2} \phi_{m}$, so that $\Delta \phi=\phi_{m}$ $-\phi_{c}<90^{\circ}-\phi_{m}$. This proves that $\phi_{m}$ is always closer to the critical angle than it is to grazing incidence $\left(90^{\circ}\right)$, consistent with Figs. 1 and 3.

15. Equation (40) can also be obtained by substituting (1 $-\rho)^{2} /(1+\rho)^{2}=-\tan ^{2}(\Delta / 2)$, when $\rho=\exp (j \Delta)$, in Eq. (4.20a) of Ref. 3 . 